\title{
Successful treatment of biliary intraepithelial neoplasia in the common bile duct via local excision: A case report
}

\author{
WEI WANG, WEI CHEN, KEWEI LI and JIAN WANG \\ Department of Biliary-Pancreatic Surgery, Ren Ji Hospital, School of Medicine, \\ Shanghai Jiao Tong University, Shanghai 200127, P.R. China \\ Received January 18, 2015; Accepted February 12, 2016
}

DOI: $10.3892 / \mathrm{ol} .2016 .4336$

\begin{abstract}
Biliary intraepithelial neoplasia (BilIN), a precursor lesion of bile duct adenocarcinomas, rarely occurs in the extrahepatic bile duct, and therefore uniform treatment guidelines are lacking. The present study reports a case of BilIN in the common bile duct (CBD) in a 77-year-old man, who presented with upper abdominal malaise without jaundice. Imaging modalities revealed a CBD luminal tumor and dilated intrahepatic and extrahepatic bile ducts. To the best of our knowledge, local excision of the lesion was performed for the first time for this type of tumor in the present study. Pathological examination gave a definite diagnosis of high-grade BilIN in the CBD. No evidence of recurrence was observed during a 3-year follow-up period. This case report suggests that local excision is able to successfully treat BilIN in the CBD.
\end{abstract}

\section{Introduction}

Biliary intraepithelial neoplasia (BilIN) is a relatively new term that is applied to flat or micropapillary precursor lesions of bile duct adenocarcinomas, which are typically located in the intrahepatic, and occasionally extrahepatic, bile ducts $(1,2)$. In surgically removed liver samples of chronic biliary disease and chronic liver disease, including hepatolithiasis, primary sclerosing cholangitis, choledochal cyst, chronic hepatitis $\mathrm{C}$ and alcoholic cirrhosis, BilIN may be identified without difficulty. However, since 2007, when the histological criteria for BilIN were confirmed, only 1 case of extrahepatic BilIN has been reported, excluding carcinoma in situ (3). The clinical knowledge of extrahepatic BilIN is limited, including incidence rates, diagnoses, treatment options, success rates, survival rates and recurrence. Uniform treatment guidelines are also lacking. The present study reports a case of BilIN in

Correspondence to: Dr Jian Wang, Department of Biliary-Pancreatic Surgery, Ren Ji Hospital, School of Medicine, Shanghai Jiao Tong University, 1630S Dongfang Road, Shanghai 200127, P.R. China E-mail: dr_wangjian@126.com

Key words: biliary intraepithelial neoplasia, common bile duct, bile duct neoplasms, precancerous conditions the common bile duct (CBD), which was treated with local resection of the lesion.

\section{Case report}

In December 2011, a 77-year-old man presented to the Department of Biliary-Pancreatic Surgery, Ren Ji Hospital (Shanghai, China) with a history of upper abdominal malaise for 10 days, which was aggravated during the previous 3 days. He had no medical or surgical history. Physical examination revealed no significant findings. The hemogram revealed the following: Hemoglobin, 124 g/l (normal range, 113-172 g/l); hematocrit, $37.3 \%$ (normal range, 33.5-50.8\%) and white blood cell count, $5.02 \times 10^{9} / 1$ (normal range, 3.70-9.20 $\times 10^{9} / 1$ ). The liver function test, including albumin, aspartate aminotransferase, alanine transaminase, alkaline phosphatase, $\gamma$ glutamyl transferase, total bilirubin and direct bilirubin, results were approximately normal. The serum level of tumor marker cancer antigen (CA) 125 was observed to be elevated at $55.8 \mathrm{U} / \mathrm{ml}$ (normal range, 0-35 U/ml). Tumor markers $\alpha$-fetoprotein, carcinoembryonic antigen, CA19-9, CA50, CA724 and CA242, and serum markers of chronic hepatitis were within the normal ranges. Abdominal ultrasonography (iU22 ultrasound system; Philips Medical Systems, Amsterdam, The Netherlands) detected dilatation of the CBD. Contrast-enhanced computed tomography (Discovery CT750 HD CT scanner; GE Healthcare, Fairfield, CT, USA) scanning revealed a distended gallbladder without calculi, and dilated intrahepatic and extrahepatic bile ducts, with a $1-\mathrm{cm}$ diameter tissue mass with enhancement in the tail-end of the CBD (Fig. 1). Magnetic resonance cholangiopancreatography (Ingenia 3.0T MR system; Philips Medical Systems) revealed a distended gallbladder without calculi and dilated intrahepatic and extrahepatic bile ducts, with a non-shadowing tissue mass in the distal end of the CBD (Fig. 2). Endoscopic retrograde cholangiopancreatography (TJF-260 duodenoscope; Olympus Corporation, Tokyo, Japan) revealed a $1-\mathrm{cm}$ diameter intraluminal filling defect in the distal CBD, and the pancreatogram produced was normal (Fig. 3).

The patient underwent laparotomy, during which sphincterotomy was performed, revealing a $1-\mathrm{cm}$ in diameter micropapillary lesion in the tail portion of the CBD. The lesion was resected along with the CBD margins, and was intraoperatively diagnosed as BilIN without malignant transformation. The lesion was considered resectable with 


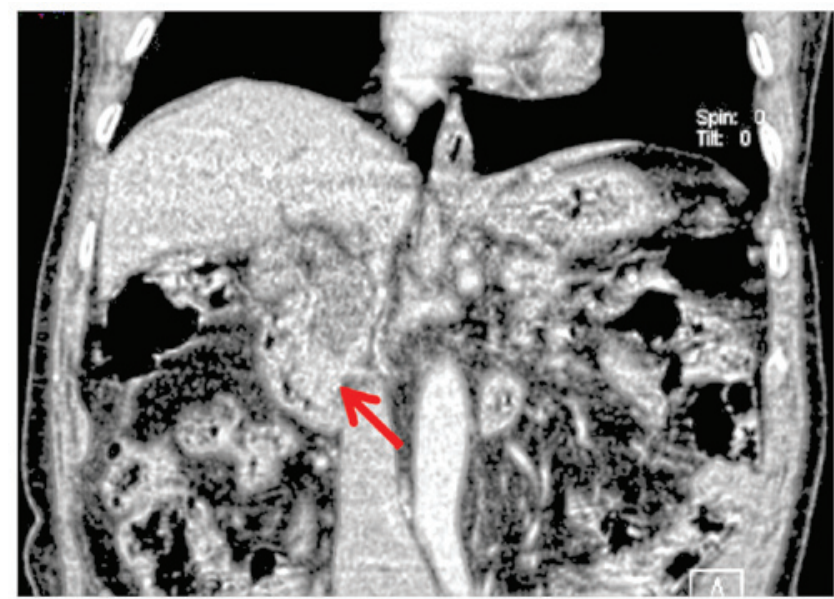

Figure 1. Contrast-enhanced computed tomography scan revealing a $1-\mathrm{cm}$ in diameter tissue mass with enhancement in the tail-end of the common bile duct (arrow).

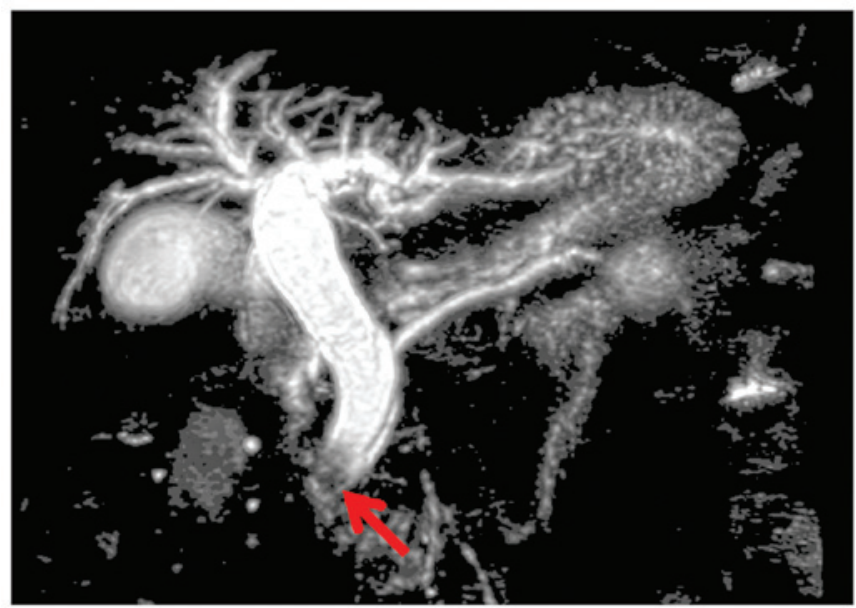

Figure 2. Magnetic resonance cholangiopancreatography showing a dis tended gallbladder without calculi, and dilated intra- and extrahepatic biliary tracts with a non-shadowing tissue mass in the distal end of the common bile duct (arrow).

safe margins and the Whipple procedure was avoided. Tumor tissue was formalin-fixed, paraffin-embedded, cut into 2-mm sections using a rotary microtome (Leica RM2235; Lecia, Mannheim, Germany) and stained with hematoxylin and eosin (Fuzhou Maixin Biotech. Co., Ltd., Fuzhou, China) for histological examination. The stained sections were observed and images were captured using a light microscope (BX43; Olympus Corporation). Pathological examination gave a definite diagnosis of high-grade BilIN in the CBD via hematoxylin and eosin staining (Fig. 4). The patient fully recovered and received no further treatment during the 3-year follow-up period, and no recurrence was identified.

\section{Discussion}

In addition to intraductal papillary neoplasm, mucinous cystic neoplasm and adenoma, BilIN is a precursor lesion of malignancy in the intrahepatic and extrahepatic bile ducts $(4,5)$. In 2005, a conceptual framework and histological criteria for

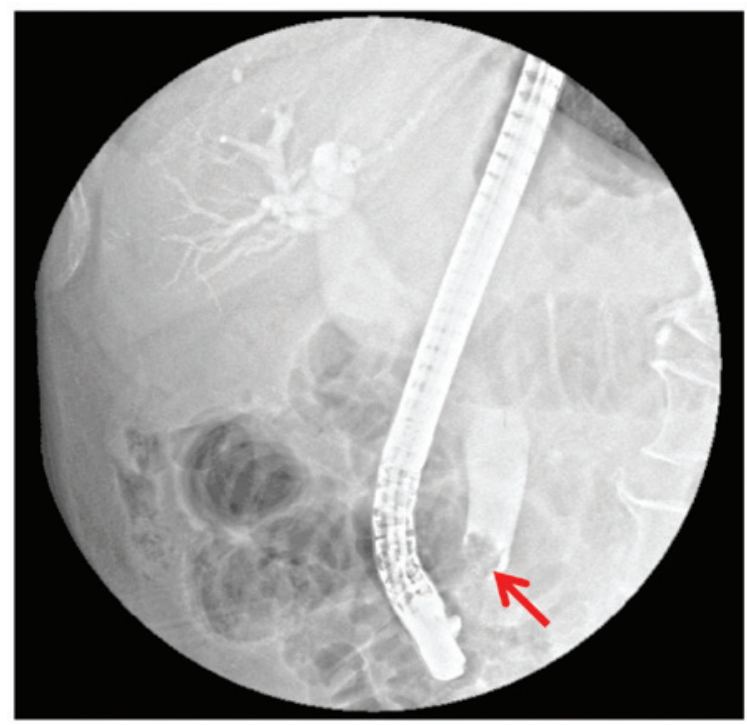

Figure 3. Endoscopic retrograde cholangiopancreatography showing a 1-cm in diameter intraluminal filling defect in the distal common bile duct (arrow).

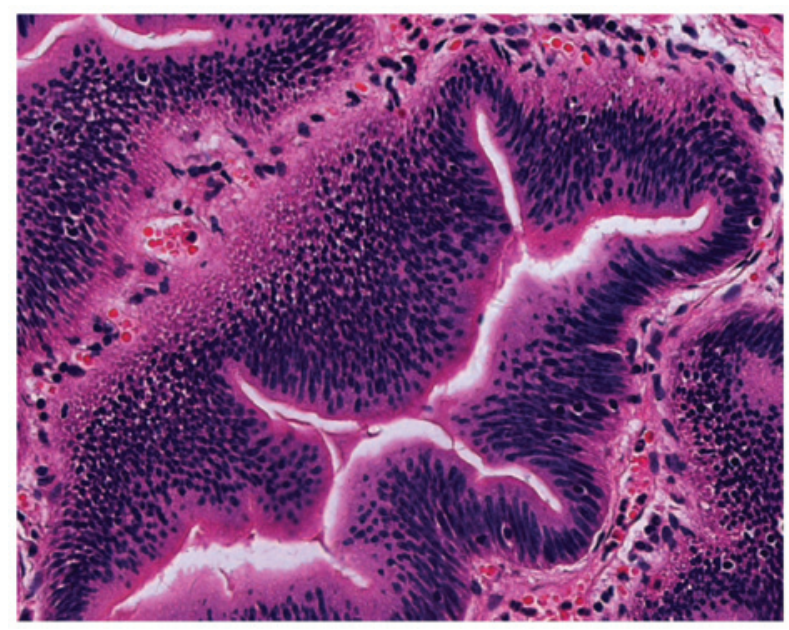

Figure 4. Histological image revealing marked nuclear dysplasia that did not reach the malignant degree of carcinoma in situ, and nuclear stratification extending into the upper third of the epithelium (hematoxylin and eosin staining; magnification, $\mathrm{x} 40$ ).

intrahepatic BilIN were proposed (6). In 2007, a consensus on the terminology and diagnostic criteria for intra- and extrahepatic BilIN was reached, and the criteria were revised (2). BilIN was classified into three grades, based on the degree of cellular and structural atypia: BilIN-1, BilIN-2 and BilIN-3. BilIN-3 additionally included carcinoma in situ (2). Based on the aforementioned criteria, the present case would be diagnosed as BilIN-2. Identification of the precursor lesion is important, since it requires minimal treatment and may serve as a good indicator of disease progression and recurrence (7).

Precursor lesions of intra- and extrahepatic bile ducts are not easily accessible due to their anatomical position, meaning it is difficult to obtain biopsy samples via endoscopic or needle biopsy (7). Furthermore, the majority of time, BilIN presents as a flat or micropapillary lesion that does not form a mass or cause bile duct obstruction (7), as was the case in the present study. Early detection or regular follow-up of 
BilIN is considerably challenging, resulting in the limited understanding of the natural course or progression of BilIN. When these tumors grow large enough to become palpable, the symptom of biliary obstruction is usually present. Common clinical presentations of patients with BilIN include jaundice, intermittent pain, dyspepsia, weight loss, nausea, vomiting, malaise and fever $(3,7,8)$. Laboratory test results typically demonstrate elevated levels of bilirubin, alkaline phosphatase and liver enzymes $(3,7,8)$. Salient imaging features include dilated and/or stenotic changes in the bile duct, occasionally with fuzzy-looking defects or endoluminal frond-like mass lesions $(3,7,8)$. The preoperative diagnosis of BilIN, and particularly the differentiation between this type of tumor and a malignant neoplasm, is challenging. Therefore, when clinicians encounter dilation and/or stenosis of the intraand/or extrahepatic bile duct with an obscure source, BilIN should be considered for differential diagnosis, particularly in patients with primary sclerosing cholangitis, choledochal cyst or hepatolithiasis, as this would assist with prompt and appropriate treatment (2). However, BilIN has a malignant potential, and surgical resection alone is only curative prior to malignant transformation; therefore accurate diagnosis is very important $(4,5)$.

Treatment options for intrahepatic BilIN range from hepatic resection to liver transplantation, according to the degree of lesion spreading $(7,8)$. However, treatment strategies for extrahepatic BilIN have rarely been reported. In a previous case report, the patient underwent pancreaticoduodenectomy (3). In the present case, the lesion was treated with local resection; however, due to BilIN's potential for malignant transformation, establishing a close monitoring protocol for any sign of disease recurrence would be a wise strategy. This would include surveillance using endoscopy and endoscopic ultrasonography, and monitoring of any changes in the hepatic functioning panel.

In conclusion, to the best of our knowledge, the present study reports the first case of extrahepatic BilIN treated using local resection. Following surgery, the patient remained in a stable condition without disease recurrence for 3 years. The present case demonstrated that local resection is a feasible treatment strategy for extrahepatic BilIN. However, further studies, in which patients are followed up for a longer time period, are required to determine the optimal treatment strategy for extrahepatic BilIN.

\section{Acknowledgements}

The authors would like to thank the Ministry of Science and Technology of the People's Republic of China (Beijing, China) (grant no. 2012BAI06B01) and the Shanghai Bureau of the Health of China (Shanghai, China) (grant nos. XBR2011035 and 20144Y0059) for their financial support.

\section{References}

1. Nakanuma Y: A novel approach to biliary tract pathology based on similarities to pancreatic counterparts: Is the biliary tract an incomplete pancreas? Pathol Int 60: 419-429, 2010.

2. Zen Y, Adsay NV, Bardadin K, Colombari R, Ferrell L, Haga H, Hong SM, Hytiroglou P, Klöppel G, Lauwers GY, et al: Biliary intraepithelial neoplasia: An international interobserver agreement study and proposal for diagnostic criteria. Mod Pathol 20: 701-709, 2007.

3. Ohtani H, Ishida H, Ito Y, Yamaguchi T and Koizumi M: Autoimmune pancreatitis and biliary intraepithelial neoplasia of the common bile duct: A case with diagnostically challenging but pathogenetically significant association. Pathol Int 61: 481-485, 2011.

4. Nakanuma Y, Sasaki M, Sato Y, Ren X, Ikeda H and Harada K: Multistep carcinogenesis of perihilar cholangiocarcinoma arising in the intrahepatic large bile ducts. World J Hepatol 1: 35-42, 2009.

5. Bickenbach K, Galka E and Roggin KK: Molecular mechanisms of cholangiocarcinogenesis: Are biliary intraepithelial neoplasia and intraductal papillary neoplasms of the bile duct precursors to cholangiocarcinoma? Surg Oncol Clin N Am 18: 215-224, vii, 2009.

6. Zen Y, Aishima S, Ajioka Y, Haratake J, Kage M, Kondo F, Nimura Y, Sakamoto M, Sasaki M, Shimamatsu K, et al: Proposal of histological criteria for intraepithelial atypical/proliferative biliary epithelial lesions of the bile duct in hepatolithiasis with respect to cholangiocarcinoma: Preliminary report based on interobserver agreement. Pathol Int 55: 180-188, 2005.

7. Jung W and Kim Bh: Biliary intraepithelial neoplasia: A case with benign biliary stricture. Korean J Hepatol 17: 328-330, 2011.

8. Aishima S, Nishihara Y, Tsujita E, Taguchi K, Soejima Y, Taketomi A, Ikeda Y, Maehara Y and Tsuneyoshi M: Biliary neoplasia with extensive intraductal spread associated with liver cirrhosis: A hitherto unreported variant of biliary intraepithelial neoplasia. Hum Pathol 39: 939-947, 2008. 